\title{
Hypothalamic self-stimulation differs as a function of anodal locus
}

\author{
RICHARD J. BODNAR \\ Department of Behavioral Physiology, New York State Psychiatric Institute, \\ New York, New York 10032 \\ and
}

\author{
SOLOMON S. STEINER, MARTIN BRUTUS, PAULA M. IPPOLITO, \\ and STEVEN J. ELLMAN \\ City College of New York, New York, New York 10031
}

\begin{abstract}
Recent reports have questioned the exclusive role of cathodal influences in the mediation of intracranial self-stimulation (ICSS) behavior. The present study examined whether variation of anodal locus affected rat hypothalamic ICSS behavior. The temporal distance (C-C interval) between succeeding monophasic pulse pairs was held constant, while the temporal distance (C-T interval) between the two first and second pulses was systematically varied in each of two anodal conditions: (1) one pole of a bipolar electrode served as cathode and the other as anode (bipolar condition), and (2) one pole of a bipolar electrode served as cathode and a cortical screw served as anode (monopolar condition). The monopolar condition produced significantly higher ICSS response rates than the bipolar condition at short $(0.5-1.0 \mathrm{msec}) \mathrm{C}-\mathrm{T}$ intervals. These data suggest that anodal placement can influence ICSS responsivity, possibly through physiological mechanisms such as size of the stimulation field or anodal hyperpolarization.
\end{abstract}

The precise identification of the physiological and anatomical substrates of intracranial self-stimulation (ICSS) behavior has been a source of debate because of the difficulty in objectively quantifying the current spread extent through tissue (Valenstein, 1964), the amount of tissue activated by the stimulation (Ranck, 1975), and whether ICSS behavior is the exclusive consequence of neural excitation at the negative (cathodal) pole as generally assumed or rather some blend of cathodal excitation and anodal inhibition (Ranck, 1975). To test the relative efficacy of cathodal and anodal influences upon ICSS behavior, our laboratory has employed a monophasic stimulation technique in which each tip of a bipolar electrode exclusively served as the cathode in one of two stimulation conditions. Response rate comparisons between the two electrode tips revealed that small $(0.1 \mathrm{~mm})$ changes in the anatomical locus of each ICSS electrode tip placement within the same animal produced marked alterations in ICSS response rate (Steiner, Bodnar, Ackermann, Nelson, \& Ellman, Note 1), which persisted regardless of whether the

This research was supported in part by a NIDA grant to S.S.S., and a CUNY grant to S.J.E. Correspondence should be sent to Dr. R. J. Bodnar, Department of Behavioral Physiology, New York State Psychiatric Institute, 722 West 168 Street, New York, New York 10032. anode was the other tip of the bipolar electrode (bipolar condition) or a screw randomly placed in the skull (monopolar condition).

In the present study, the role of anodal locus upon hypothalamic ICSS poststimulation excitability curves was determined through the use of the monophasic pulse-pair stimulation technique. In this technique, stimulation consists of a train of paired monophasic rectangular electrical pulses; the first pulse of each pair is designated the $C$ pulse, while the second pulse is designated the $\mathrm{T}$ pulse. The temporal distance between succeeding $C$ pulses is called the $C-C$ interval, while the temporal distance between $\mathrm{C}$ and $T$ pulses is called the C-T interval. If the C-C interval is held constant while the C-T interval is systematically varied, medial forebrain bundle ICSS rates change as a function of this variation (Deutsch, 1964; Gallistel, 1969; German \& Holloway, 1972; Rolls, 1971; Smith \& Coons, 1970; Szabo, Lenard, \& Kosaras, 1974; Yeomans, 1975). Trains of stimulation with long C-T intervals (50 msec or longer) elicit lower ICSS rates at the same current level than stimulation trains with shorter C-T intervals $(1.5-50 \mathrm{msec})$. However, when the C-T intervals are shortened below a critical value (0.5-1.2 msec), ICSS response rates sharply diminish to a value elicited by stimulation comprising only $\mathrm{C}$ pulses ( $\mathrm{T}$ pulses omitted), suggesting that at very short $\mathrm{C}$ - $\mathrm{T}$ intervals, the 
$T$ pulses are rendered ineffective. The response rate reduction noted at very short $\mathrm{C}$ - $\mathrm{T}$ intervals has been attributed to either the characteristic refractory limitations of the axonal elements situated beneath the electrode tip (Deutsch, 1964; Gallistel, 1969; German \& Holloway, 1972; Rolls, 1971; Smith \& Coons, 1970; Yeomans, 1975) or alternatively due to synaptic changes (Szabo, 1973; Szabo et al., 1974).

The present study examined whether alterations in anodal locus contributed to the inability of short C-T intervals to sustain ICSS behavior. If these response rate reductions are exclusively mediated by cathodal refractory limitations, then changes in anodal locus should not alter ICSS poststimulation excitability curves. However, if anodal factors are involved in ICSS behavior, as has been previously suggested (Ranck, 1975), then alterations in anodal locus should correspondingly affect ICSS poststimulation excitability curves. Therefore, each animal was tested for ICSS responsivity over a range of different C-T intervals under bipolar and monopolar stimulation conditions.

\section{METHOD}

Twelve male albino Holtzman Sprague-Dawley rats (350-500 g) were anesthetized with Chloropent (Fort Dodge Laboratories; $1 \mathrm{ml} / \mathrm{kg}$ ) and stereotaxically (Kopf) implanted with a bipolar electrode (Plastic Products) aimed at the hypothalamus (HYP). Each bipolar electrode was insulated except at the tips, which were aligned mediolaterally perpendicular to the midsagittal plane; each tip was separated from the other by a maximum of $0.1 \mathrm{~mm}$. Two stainless steel cortical screws were attached to the skull and electrically connected to an electrode plug. These screws, which were randomly assigned placement on the skull, could each serve as separate anodal indifferents. With the incisor bar set at - $5 \mathrm{~mm}$, HYP coordinates were $4.2-4.4 \mathrm{~mm}$ posterior to bregma, $1.5 \mathrm{~mm}$ lateral to the sagittal suture, and $8.7 \mathrm{~mm}$ from the top of the skull.

Ten days after surgery, each animal was shaped to barpress for brain stimulation in an operant conditioning chamber on a continuous reinforcement schedule. Reinforcements were $700 \mathrm{msec}$ trains of negatively going, capacitance-coupled, monophasic rectangular pulses of electrical stimulation delivered to an animal's electrode site. Any responses made during a stimulation train were ineffective. The stimulator was constructed from Digi-Bit solid state logic circuitry (BRS/LVE) which allowed the experimenter to set the pulse duration $(0.1 \mathrm{msec})$ and the $\mathrm{C}-\mathrm{C}$ interval $(20$ or $30 \mathrm{msec}$ ) and independently manipulate whether or not the $T$ pulses were delivered, and if delivered, the C-T interval. Waveform, C-C interval, stimulus intensity and pulse and train durations were continuously monitored by observing on a cathode ray oscilloscope the voltage drop across a 100 -ohm resistor in series with the animal.

The animals were shaped for a maximum of 15 daily sessions; the pole of the bipolar electrode which elicited the higher response rates in preliminary testing (see Steiner et al., Note 1) served as the cathode. For each electrode site, the lowest intensity was chosen which yielded consistently high ICSS response rates at a C-T interval of $5.0 \mathrm{msec}$, but less than $10 \mathrm{responses} / \mathrm{min}$ when the $T$ pulses were omitted. The criterion intensities across animals varied from 200 to $600 \mu \mathrm{A}$. For nine animals, the same criterion intensity was tested for both experimental conditions. Of the remaining three animals, two were tested at a lower intensity in the monopolar condition while one was tested at a lower intensity in the bipolar condition. These intensities were comparable to or lower than intensities used in other studies employing monophasic pulse pairs of brief $0.1-\mathrm{msec}$ duration (Deutsch, 1964; Gallistel, 1969; German \& Holloway, 1972; Rolls, 1971; Smith \& Coons, 1970; Szabo, 1973). It should be noted that the number of microcoulombs delivered in this stimulation technique is substantially less than that delivered by traditional $60-\mathrm{Hz}$ sinusoidal stimulation.

Every day, each animal barpressed for stimulation delivered to its electrode site during a 72-min session, which was divided into nine 7-min periods with a 1-min time-out between each period, during which the $\mathrm{C}-\mathrm{T}$ interval was changed. Eight C-T intervals $(0.5,0.8,1.0,1.2,1.5,2.0,3.0$, and $5.0 \mathrm{msec})$ and a condition in which the $T$ pulses were omitted were randomly presented in each of 9 days in a Latin square design. The mean response rate over the last $5 \mathrm{~min}$ of each 7 -min period constituted the dependent variable. Data from the first 2 min of each 7 -min period were excluded from the analysis of experimental effects and revealed that the Latin square design adequately controlled for order and carry-over effects.

A C-T interval/response rate function, averaged over the 9-day paradigm was determined for each animal for two conditions: (1) when one pole of the bipolar electrode served as cathode and the other pole served as anode through the entire period of stimulation (bipolar condition), and (2) when the same cathodal pole of the bipolar electrode was tested in conjunction with a cortical screw serving as anode (monopolar condition). These conditions were tested in counterbalanced order across animals.

After completion of the experiment, all animals were overdosed with Chloropent and perfused through the heart with normal saline followed by $10 \%$ Formalin. Serial frozen sections were stained with luxol fast blue and cresyl violet (Kluver \& Barrera, 1953) and electrode loci determined by microscopic examination of the sections.

\section{RESULTS}

All 12 electrode placements supported ICSS behavior and were localized in the HYP (Figure 1). The criterion intensity for each animal did not significantly differ for bipolar and monopolar conditions. No significant differences between bipolar and monopolar ICSS response rates elicited when the $T$ pulses were omitted were observed $(p>.05)$; these rates were always less than 10 responses/min. To determine the relative effectiveness of the $T$ pulses at different C-T intervals for each animal in each condition, response rates elicited when the $T$ pulses were omitted were subtracted from response rates elicited at all of the C-T intervals. With these scores, a randomizedblock, repeated-measure three-way analysis of variance was performed with stimulation condition, C-T interval, and order of testing as main effects.

ICSS response rates were significantly higher, $F(1,165)=5.76, p<.05$, in the monopolar condition than in the bipolar condition. ICSS response rates were also significantly different, $F(7,165)=$ $27.20, \mathrm{p}<.01$, across the C-T intervals. No significant order effect was observed $(p>.05)$; therefore, the higher rates elicited in the monopolar condition were not contingent upon whether that condition was tested before or after the bipolar condition. Further- 


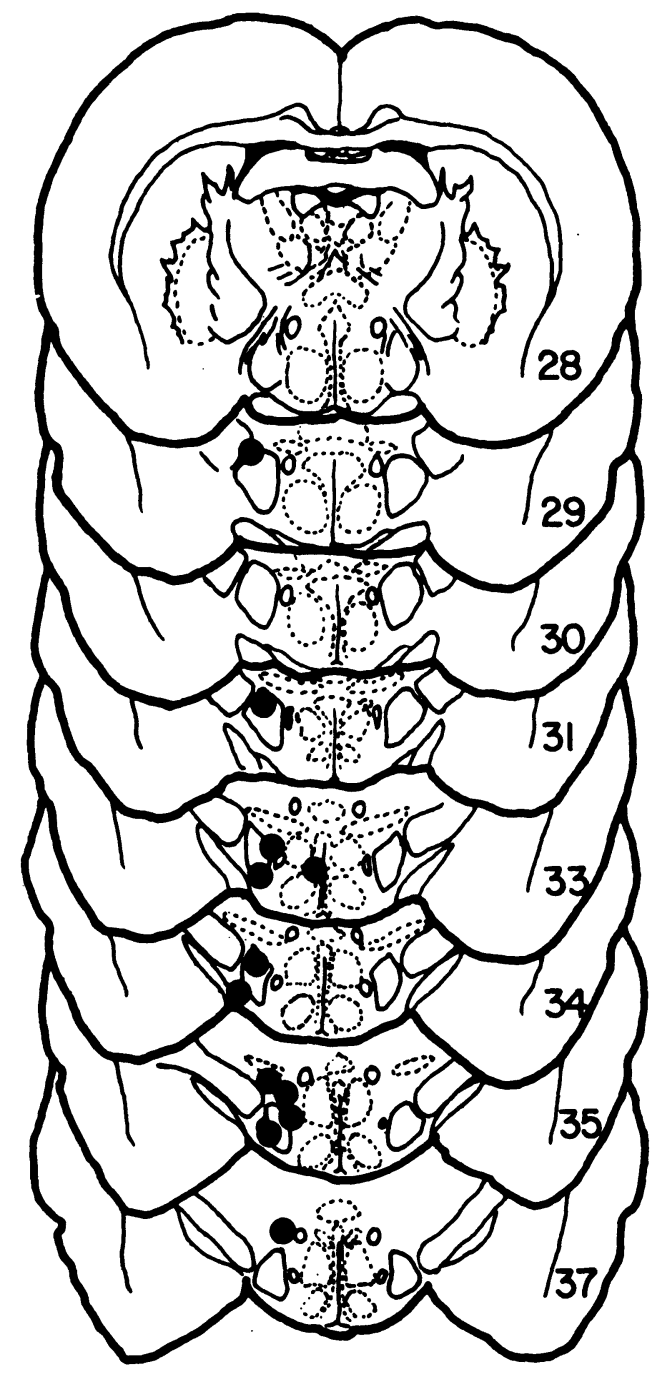

Figure 1. Schematic representation of the 12 hypothalamic electrode placements. The sections are derived from Konig and Klippel (1963).

more, no significant interactions between main effects were observed. Figure 2 displays the variation in response rates as a function of C-T interval for the monopolar and bipolar conditions. A posteriori Tukey comparisons revealed that the monopolar condition produced significantly higher response rates than the bipolar condition at the short $C-T$ intervals, including, $0.5 \mathrm{msec}[\mathrm{t}(11)=2.34, \mathrm{p}<.05], 0.8 \mathrm{msec}$ $[\mathrm{t}(11)=3.05, \mathrm{p}<.05]$, and $1.0 \mathrm{msec}[\mathrm{t}(11)=2.61$, $\mathrm{p}<.05]$. In contrast, response rates elicited by the bipolar and monopolar conditions were similar at C-T intervals of $1.2 \mathrm{msec}$ and longer.

Given that response rates were significantly higher in the monopolar condition at short C-T intervals, linear regression analyses of ICSS response rate for each stimulation condition were carried out for the
C-T intervals between 0.5 and $1.5 \mathrm{msec}$ and for the $\mathrm{C}$-T intervals between 1.5 and $5.0 \mathrm{msec}$. Table 1 displays the regression equations and coefficients for monopolar and bipolar conditions. At short C-T intervals, the intercept of the monopolar condition was significantly higher $[\mathrm{t}(11)=4.71, \mathrm{p}<.001)]$ than the bipolar condition. Also, the slope in the monopolar function was shallower, but not significantly so. These data indicate that monopolar response rates at the shortest C-T interval tested are considerably higher than their bipolar counterparts and hence need a less accelerated rise to the peak responding at the longer $\mathrm{C}-\mathrm{T}$ intervals. At the longer $\mathrm{C}-\mathrm{T}$ intervals, the intercepts of the two stimulation conditions did not differ, demonstrating the equipotent effectiveness of both stimulation conditions to support optimal responding. However, the slope of the monopolar function was significantly shallower $[\mathrm{t}(11)=$ $3.11, \mathrm{p}<.01$ ], indicating that even at the longer $(1.5-5.0 \mathrm{msec}) \mathrm{C}-\mathrm{T}$ intervals, the bipolar function was still in a more accelerated rise to peak responding, while the monopolar function reached an asymptote.

\section{DISCUSSION}

Anodal placements distant from a HYP cathodal source markedly enhance ICSS responsivity at those C-T intervals which normally fail to support ICSS behavior when cathodal and anodal sources are adjacent. Several hypotheses might account for the

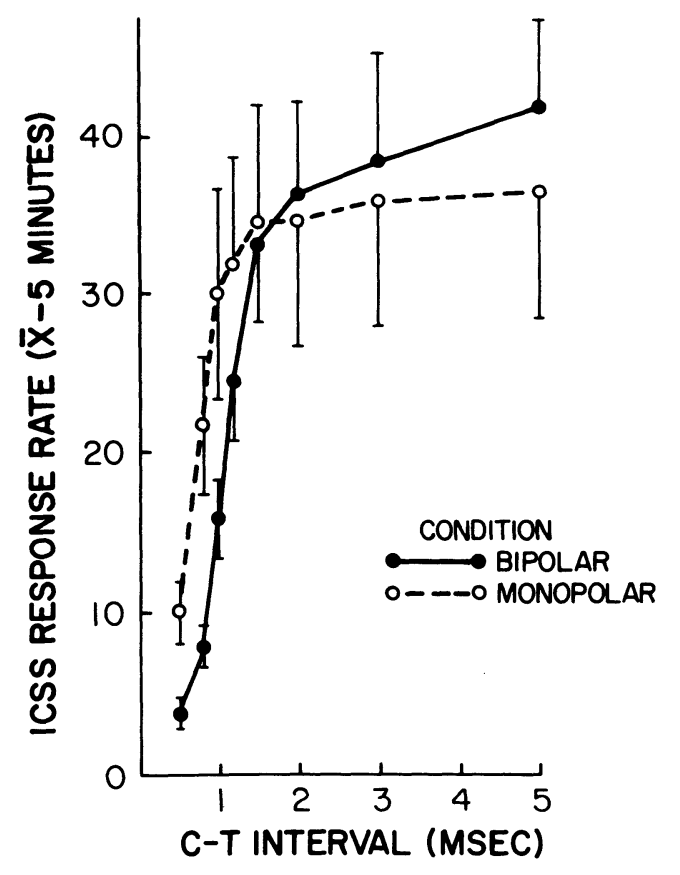

Figure 2. Mean ICSS response rate ( \pm S.E.M.) as a function of $C-T$ interval for the bipolar and monopolar stimulation conditions. 
Table 1

Regression Equations and Coefficients $\left(\mathrm{r}^{2}\right)$ for the Monopolar and Bipolar Conditions

\begin{tabular}{|c|c|c|c|c|}
\hline \multirow{2}{*}{$\begin{array}{c}\text { C-T Interval } \\
(\mathrm{msec})\end{array}$} & \multicolumn{2}{|c|}{ Monopolar } & \multicolumn{2}{|c|}{ Bipolar } \\
\hline & Equation & $r^{2}$ & Equation & $\mathrm{r}^{2}$ \\
\hline $\begin{array}{r}.5-1.5 \\
1.5-5.0\end{array}$ & $\begin{array}{l}y=25.38 x+.04 \\
y=.57 x+33.68\end{array}$ & $\begin{array}{l}.880 \\
.909\end{array}$ & $\begin{array}{l}y=31.58 x-14.69 \\
y=2.27 x+30.86\end{array}$ & $\begin{array}{l}.976 \\
.9284\end{array}$ \\
\hline
\end{tabular}

greater efficacy of monopolar stimulation at these pulse pair intervals.

First, a larger stimulation field might be produced during monopolar stimulation as compared to bipolar stimulation because the distance between cathodal and anodal sources of stimulation is greater. This larger field could hypothetically produce more neural paths in the monopolar condition through which physiologically active (cathodal) current could be passed. This, in turn, would activate more rewardrelevant neurons beneath the electrode tip at each C-T interval. The present data agree with such a hypothesis, since near-optimal ICSS rates were attained by a C-T interval of $1.0 \mathrm{msec}$ in the monopolar condition as compared to $1.5 \mathrm{msec}$ in the bipolar condition. This hypothesis would predict such an increase when the stimulated ICSS site is adjacent to other ICSS sites. ICSS can be elicited from many HYP structures, including the medial forebrain bundle, fornix, far-lateral hypothalamus, zona incerta, and, to a lesser extent, the medial and periventricular nuclei (see review, German \& Bowden, 1974). Therefore, if a cathodal source, localized in one HYP locus, is paired with an anodal source distant from the HYP, the resultant stimulation field would comprise a number of ICSS loci which would be activated by the stimulation and thereby increase ICSS rates at short C-T intervals. However, it should be noted that this hypothesis does not explain why response rates at very short $\mathrm{C}-\mathrm{T}$ intervals $(0.5$, $0.8 \mathrm{msec}$ ) did not attain peak levels in the monopolar condition or why both monopolar and bipolar rates were similar when only the $C$ pulses were delivered. Such outcomes would be expected if the larger stimulation field hypothesis was exclusively correct.

A second explanation for the greater efficacy of monopolar stimulation at short C-T intervals is that bipolar stimulation produces greater anodal hyperpolarization beneath the electrode tip. Delivery of an initial pulse of a pulse pair would hypothetically increase the neural action potential threshold via anodal hyperpolarization and decrease the probability that a subsequent pulse would effect depolarization of the stimulation field. This hypothesis would assume that if the threshold to activate rewardrelevant axonal elements is increased, then the ongoing behavior subserved by these elements should be reduced. If this hypothesis is correct, one would predict that the stimulation's hyperpolarizing effects would be more acute in the bipolar condition in producing an inhibition in ICSS response rate, since cathodal and anodal fields are adjacent. At short $C$-T intervals, the arrival of the $T$ pulse of each pulse pair would coincide with a hyperpolarized field produced by the preceding $C$ pulse rendering the $T$ pulse less capable of effecting depolarization of the rewardrelevant neurons necessary to sustain ICSS behavior. However, as the C-T interval is increased in the bipolar condition, the additional temporal spacing between $C$ and $T$ pulses would hypothetically allow these reward-relevant elements to recover to resting levels before delivery of the subsequent $\mathrm{T}$ pulses, thereby effecting depolarization and supporting ongoing ICSS behavior. Essentially, such results were obtained in the bipolar condition. However, one would predict that uniform responding should occur across all $\mathrm{C}-\mathrm{T}$ intervals in the monopolar condition, unless the anodal field produced by the cortical screw is large enough to hyperpolarize pertinent neurons stimulated by the cathode.

The elucidation of these hypothesized mechanisms does not imply that they are mutually exclusive of one another, or that other physiological events such as the refractory characteristics of neurons are not important in producing the differences noted between monopolar and bipolar stimulation conditions. Based on the data of the present study, however, an exclusive role for the refractory characteristics of neurons in mediating the occurrence of ICSS behavior would be doubtful because the cathodal source in both monopolar and bipolar conditions is the same and would be expected to display similar functions.

\section{REFERENCE NOTE}

1. Steiner, S. S., Bodnar, R. J. Ackermann, R. F., Nelson, W. T., \& Ellman, S. J. Intracranial self-stimulation site specificity: The myth of current spread. Submitted for publication.

\section{REFERENCES}

DEUTSCH, J. A. Behavioral measurement of the neural refractory period and its application to intracranial self-stimulation. Journal of Comparative and Physiological Psychology, 1964, 58, 1-9.

Gallistel, C. R. The incentive of brain-stimulation reward. Journal of Comparative and Physiological Psychology, 1969, 69. 713-721. 
German, D. C., \& Bowden, D. M. Catecholamine systems as the neural substrate for intracranial self-stimulation. Brain Research, 1974, 73, 381-419.

German, D. C., \& Holloway, F. A. Behaviorally determined neurophysiological properties of MFB self-stimulation fibers. Physiology and Behavior, 1972, 9, 823-829.

Kluver, H., \& BarRera, E. A method for combined staining of cells and fibers in the nervous system. Journal of Neuropathology and Experimental Neurology, 1953, 12, 400-403.

KönIG, J. F. R., \& KLIPPEL, R. A. The rat brain: A stereotaxic atlas. Baltimore: Williams \& Wilkins, 1963.

RANCK, J. B. Which elements are excited in electrical stimulation of mammalian central nervous system: A review. Brain Research, 1975, 98, 417-440.

Rolss, E. T. Absolute refractory period of neurons involved in MFB self-stimulation. Physiology and Behavior, 1971, 7, 311-315.

Smith, N., \& Coons, E. E. Temporal summation and refractori- ness in hypothalamic reward neurons as measured by selfstimulation behavior. Science, 1970, 169. 782-785.

SzABo, I. Path neuron system of medial forebrain bundle as a possible substrate for hypothalamic self-stimulation. Physiology and Behavior, 1973, 10, 315-328.

Szabo, I., Lenard, L., \& Kosaras, B. Drive decay theory of self-stimulation: Refractory periods and axon diameters in hypothalamic reward loci. Physiology and Behavior, 1974. 12. 329-343.

Valenstein, E. S. Problems of measurement and interpretation with reinforcing brain stimulation. Psychological Review, 1964 . 71, 415-437.

Yeomans, J. S. Quantitative measurement of neural poststimulation excitability with behavioral methods. Physiology and Behavior, 1975, 15, 593-602.

(Received for publication August 17, 1977; revision accepted November 22, 1977.) 